\title{
Detection of Regional Wall Motion Abnormalities in Compressed Sensing Cardiac Cine Imaging
}

\author{
Juliane Goebel ${ }^{1}$, Felix Nensa1, Haemi Schemuth1', Stefan Maderwald ${ }^{2}$, Harald H. Quick ${ }^{2,3}$, \\ Thomas Schlosser ${ }^{1}$, Kai Nassenstein ${ }^{1}$
}

${ }^{1}$ Department of Diagnostic and Interventional Radiology and Neuroradiology, University Hospital Essen, Essen, Germany ${ }^{2}$ Erwin L. Hahn Institute for Magnetic Resonance Imaging, University of Duisburg-Essen, Essen, Germany

${ }^{3}$ High Field and Hybrid MR Imaging, University of Duisburg-Essen, Essen, Germany

Email: *Juliane.Goebel@uk-essen.de

How to cite this paper: Goebel, J., Nensa, F., Schemuth, H., Maderwald, S., Quick, H.H., Schlosser, T. and Nassenstein, K. (2018) Detection of Regional Wall Motion Abnormalities in Compressed Sensing Cardiac Cine Imaging. World Journal of Cardiovascular Diseases, 8, 277-287.

https://doi.org/10.4236/wjcd.2018.86027

Received: April 20, 2018

Accepted: June 9, 2018

Published: June 12, 2018

Copyright $\odot 2018$ by authors and Scientific Research Publishing Inc. This work is licensed under the Creative Commons Attribution International License (CC BY 4.0).

http://creativecommons.org/licenses/by/4.0/

\begin{abstract}
Background: Recently faster cardiac magnetic resonance (CMR) cine sequences basing on k-t compressed sensing have been developed. Purpose: To compare two compressed sensing CMR sequences-one in breath-hold technique and one during free breathing-with the standard SSFP sequence with respect to regional left ventricular function assessment. Material and Methods: Left ventricular short-axis stacks of two compressed sensing sequences in breath-hold technique (sparse_HB) and during free breathing (sparse_FB; both spatial resolution, $1.8 \times 1.8 \times 8 \mathrm{~mm}^{3}$ ) and a standard SSFP cine sequence (spatial resolution, $1.9 \times 1.9 \times 8 \mathrm{~mm}^{3}$ ) were acquired in 50 patients on a $1.5 \mathrm{~T}$ MR system. Regional wall motion abnormalities (RWMA) were rated qualitatively (normal/hypo-/a-/dyskinesia) by two experienced readers in consensus for all cardiac segments (American Heart Association's segment model) and sequences. RWMA detection rates were compared between sequences by kappa statistic. Results: In 13 patients, RWMA were detected in at least one cardiac segment. The RWMA detection rates were similar between CMR sequences (hypokinesia, $7.2 \%$ to $7.9 \%$; akinesia, $0.8 \%$ to $1.3 \%$; dyskinesia $0.3 \%$ to $0.4 \%$ ) and kappa statistics revealed an almost perfect agreement in RWMA detection between both sparse and the standard SSFP sequence (standard versus sparse_HB: kappa, $0.918, p$ value, $<0.001$; standard versus sparse_FB: kappa, 0.868, $p$ value, $<0.001)$. Conclusion: Compressed sensing cine CMR acquired during breath-hold or free-breathing allows reliable RWMA detection, thus, might alternatively be used in cine CMR for regional left ventricular function assessment.
\end{abstract}




\section{Keywords}

Cardiac Imaging Techniques, Cine Magnetic Resonance Imaging, Cardiac Muscle, Cardiac Volume, Myocardial Contraction

\section{Introduction}

Cardiac magnetic resonance imaging (CMR) is an established imaging tool in the diagnostic workup of patients with suspected heart disease and plays an important role in risk stratification (e.g. in coronary artery disease or myocarditis) and non-invasive therapy monitoring [1] [2] [3]. Due to continuous technical progress in CMR sequence design, hopefully the time consuming acquisition of standard breath-hold steady-state free precession (SSFP) cine sequences might be replaced by faster alternatives, which allow either a significant reduction in breath-hold times or even image acquisition during free breathing, which would be extremely helpful in patients with limited breath-hold capability. In this context, the recently developed k-t compressed sensing sequence technique with parallel imaging and iterative reconstruction seems very promising [4] [5] [6] [7]. Provided that special conditions regarding image properties, aliasing artifacts, and image reconstruction are fulfilled, this technique allows a considerable acceleration of CMR data acquisition due to noticeable k-space under sampling [4] [5]. Until now, several slightly different SSFP sequences basing on this new technical approach have been evaluated by different groups with the main focus on global left ventricular (LV) function [8]-[13]. Regarding global LV function, the overall consensus of these studies was that compressed sensing cine SSFP sequences are as reliable as conventional cine SSFP imaging. Despite the fact that regional wall motion abnormality (RWMA) detection is also of high clinical relevance in many settings (e.g. assessment of ischemic heart disease, high-dose dobutamine stress CMR, where an ischemia is defined as a stress-induced new or aggravated RWMA [14] [15] [16] [17] [18]), only limited data exists concerning regional wall motion assessment by compressed sensing cine imaging.

Thus, the aim of the present study was to compare two different compressed sensing CMR sequences-one acquired in breath-hold technique, with reduced breath-hold times and one during free breathing-with the current standard SSFP sequence with the focus on regional left ventricular function assessment.

\section{Material and Methods}

Prospective analysis and use of data was approved by the local ethic committee. All included patients gave written informed consent for CMR examination and study participation.

\subsection{Cardiovascular Magnetic Resonance Imaging}

All CMR scans were performed on a 1.5-Tesla system (Magnetom Aera, Siemens 
Healthcare, Erlangen, Germany). Three stacks of short-axis slices covering the complete left ventricle were acquired in every patient using the following sequences: [A] retrospectively ECG-gated, segmented cine steady-state free precession sequence in breath-hold technique (standard SSFP; TR: $44.54 \mathrm{~ms}$; TE: 1.1 ms; matrix: $192 \times 156$; FOV: $370 \times 301 \mathrm{~mm}^{2}$; flip angle: $59^{\circ}$; 17 segments; 25 calculated phases; spatial resolution: $1.9 \times 1.9 \times 8 \mathrm{~mm}^{3}$; bandwidth: $930 \mathrm{~Hz} / \mathrm{px}$; median breath-hold time (for acquisition of all short-axis slices): $130 \mathrm{sec}$ ), [B, C] prospectively ECG-triggered segmented compressed sensing cine SSFP sequence in breath-hold technique (B, sparse_HB; median breath-hold time (for acquisition of all short-axis slices): $21 \mathrm{sec}$ ) and during free breathing (C, sparse_FB) (sparse_HBand sparse_FB: TR: $39,75 \mathrm{~ms}$; TE: $1.1 \mathrm{~ms}$; matrix: $224 \times 146$; FOV: $400 \times 300 \mathrm{~mm}^{2}$; flip angle: $60^{\circ}$; 15 segments; spatial resolution: $1.8 \times 1.8 \times 8$ $\mathrm{mm}^{3}$; vendor provided sparse acceleration factors, A (defined as the acceleration factor in the central part of the k-space): 3 and B (defined as the acceleration factor in the k-space periphery): 14; number of iterations: 80; bandwidth: 893 $\mathrm{Hz} / \mathrm{px})$.

\subsection{Assessment of Regional Wall Motion Abnormalities and Left Ventricular Volumetry}

Analysis for RWMA was performed in consensus by two experienced readers (CMR experience $>12$ years and $>6$ years). Presence and severity of RWMA were evaluated visually and graded as "normal", "hypokinesia", "akinesia", or "dyskinesia" in all CMR sequences and in all cardiac segments, except for segment 17 (in accordance to the American Heart Association's segmental model) [14] [19]. Thus, a total of 800 cardiac segments in each CMR sequence were analyzed (50 patients with 16 analyzed cardiac segments each).

Left ventricular volumetry was performed in all patients and CMR sequences by one reader (CMR experience $>6$ years) using the Argus software (Siemens Healthcare, Erlangen, Germany; employed standard values based upon [20]). Because excellent inter-rater agreement for left ventricular volumes has been repeatedly reported for segmented compressed sensing cine SSFP sequences in breath-hold technique and during free breathing, we waived this subanalysis [10] [11] [13]. The papillary muscles and trabecullae were attributed to the ventricular cavity, and the most basal short-axis slice to be included into volumetry was defined by having at least $270^{\circ}$ of the chamber circumference surrounded by visible myocardium [21] [22].

\subsection{Statistical Analysis}

For statistical analysis MedCalc (version 12.3.0.0, MedCalc Software, Mariakerke, Belgium) and SPSS software package (version 19.0, IBM, Armonk, NY, USA) were used. Testing for normal distribution was performed by D'AgostinoPearson test. Normally distributed data are presented as mean \pm standard deviation, otherwise medians and interquartile ranges are given. To analyze for dif- 
ferences in RWMA detection between the three employed CMR sequences kappa statistic was performed and interpreted as proposed by Landis and Koch [23]. Comparison of volumetric data between the CMR sequences was done by Wilcox on test, and inter-rater agreement was assessed by Bland-Altman analysis. A $\mathrm{p}$ value less than 0.05 was considered statistically significant.

\section{Results}

\subsection{Patients}

Fifty consecutive unselected patients (17 female, 33 male) referred for clinical CMR examination and willed to participate in the present study were examined. Patients were referred to CMR for suspected myocarditis $(\mathrm{n}=14)$, pericarditis ( $\mathrm{n}$ $=1)$, cardiac infarction $(n=5)$, cardiomyopathy $(n=12)$, congenital heart disease $(n=2)$, cardiac tumor $(n=1)$, or unclear reduction of heart output or dysrhythmia $(\mathrm{n}=15)$. Mean patient age was $41.5 \pm 20.2$ years (range: $8-77$ years). Median weight was $75 \mathrm{~kg}$ (interquartile range: $27 \mathrm{~kg}$; range: 30 - $152 \mathrm{~kg}$ ), median height $175 \mathrm{~cm}$ (interquartile range: $12 \mathrm{~cm}$; range: $130-195 \mathrm{~cm}$ ), and median body mass index $24.8 \pm 7.2 \mathrm{~kg} / \mathrm{m}^{2}$ (range: $13.1-51.4 \mathrm{~kg} / \mathrm{m}^{2}$ ). The median heart rate was 68 beats/minute (interquartile range: 16 beats/ minute; range: 46 - 113 beats/minute).

\subsection{Regional Wall Motion Abnormalities (RWMA)}

In all 50 patients well analyzable data sets of all three CMR sequences were acquired (Figure 1 and Figure 2). In 37 patients no RWMA was detected. In 13 patients RWMA was found in at least one cardiac segment (Figure 2). Comparison of the RWMA detection rate between CMR sequences showed quite equal detection rates ranging from $7.2 \%$ to $7.9 \%$ for hypokinesia, $0.8 \%$ to $1.3 \%$ for akinesia, and $0.3 \%$ to $0.4 \%$ for dyskinesia (Table 1). Kappa statistics revealed an almost perfect agreement in RWMA detection between standard SSFP and sparse_HB (Cohens kappa, 0.918, $p$ value, $<0.001$ ), between standard SSFP and sparse_FB (Cohens kappa, $0.868, p$ value, $<0.001$ ), and between sparse_HBand sparse_FB (Cohens kappa, $0.880, p$ value, $<0.001$ ).

\subsection{Left Ventricular Volumetry}

Left ventricular volumetric values of all three CMR sequences are presented in Table 2. Comparing standard SSFP and sparse_HB, small, but significant median

Table 1. Detection rates of regional wall motion abnormalities given in absolute numbers and percent values (in brackets) for the three CMR sequences.

\begin{tabular}{ccccc}
\hline & normokinesia & hypokinesia & akinesia & dyskinesia \\
\hline standard SSFP & $729(91.1 \%)$ & $63(7.9 \%)$ & $6(0.8 \%)$ & $2(0.3 \%)$ \\
sparse_HB & $725(90.6 \%)$ & $62(7.8 \%)$ & $10(1.3 \%)$ & $3(0.4 \%)$ \\
sparse_FB & $731(91.4 \%)$ & $58(7.2 \%)$ & $9(1.1 \%)$ & $2(0.3 \%)$ \\
\hline
\end{tabular}

SSFP, steady-state free precession sequence; sparse, compressed sensing sequence; HB, breath-hold; FB, free breathing. 


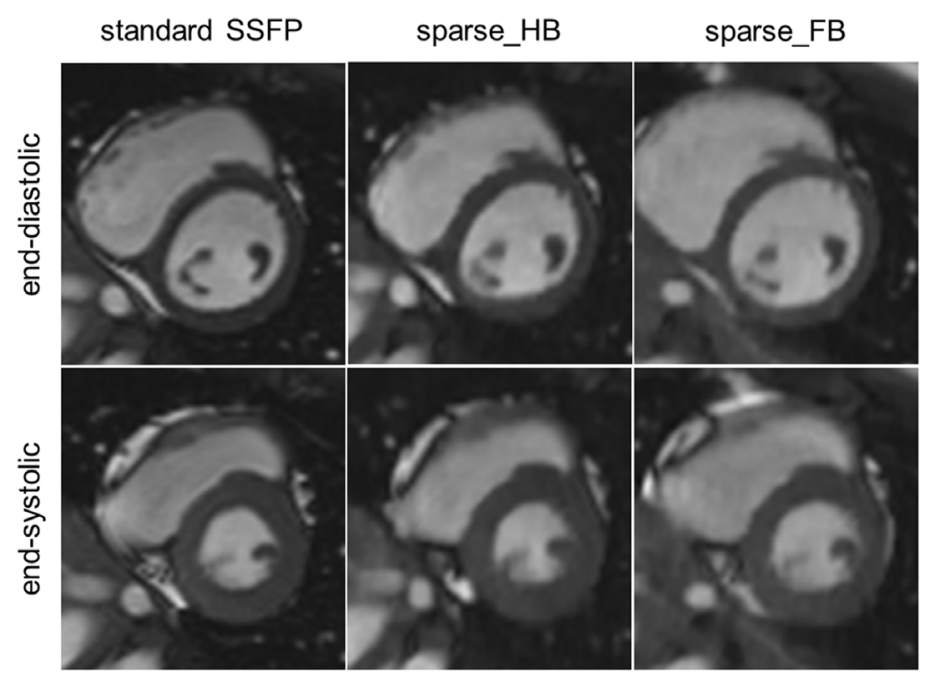

Figure 1. Midventricular short-axis slices in end-diastole and -systole in a 14-year-old male with history of anthracycline-based chemotherapy for acute lymphatic leukemia reveal an unimpaired global and regional left ventricular function: in all three employed CMR sequences excellent imaging details and blood-myocardium contrast can be seen (standard SSFP sequence; compressed sensing sequence in breath-hold technique (sparse_HB); compressed sensing sequence during free breathing (sparse_FB)).

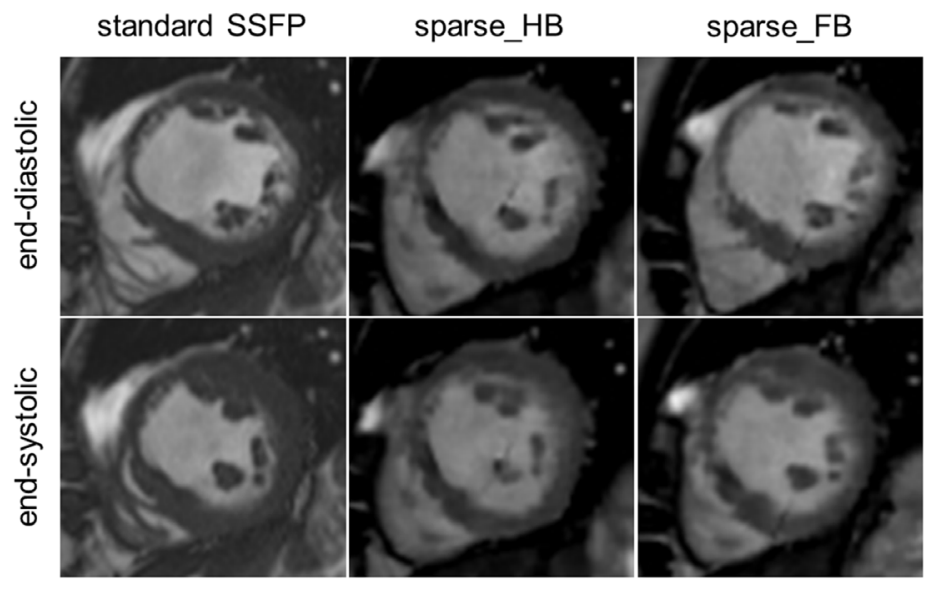

Figure 2. Short-axis slices of a 65-year-old woman with history of transmural myocardial infarction: in all three CMR sequences (standard SSFP; sparse in breath-hold (sparse_HB); sparse during free breathing (sparse_FB)) an anteroseptal and anterior akinesia is clearly detectable.

differences were found for EDV (difference of median, $8 \mathrm{ml}$; $p$ value $<0.001$ ), SV (difference of median, $8 \mathrm{ml} ; p$ value $<0.001$ ), and $\mathrm{EF}$ (difference of median, $1 \%$; $p$ value, 0.016 ), but not for ESV ( $p$ value, 0.198 ). Comparing standard SSFP and sparse_FB, small, but significant median differences were found for ESV (difference of median, $4 \mathrm{ml} ; p$ value $<0.001$ ), SV (difference of median, $4 \mathrm{ml} ; p$ value, 0.004 ), and EF (difference of median, $2 \%$; $p$ value $<0.001$ ), but not for $\operatorname{EDV~(~} p$ value, 0.817). These findings were confirmed by the Bland-Altman analysis (Table 3, Figure 3), where an overall good agreement in volumetric values between the standard SSFP sequence and both sparse sequences was found. 
Table 2. Volumetric values (presented as median and interquartile range (in brackets)) of all 50 investigated patients comparing the three CMR sequences.

\begin{tabular}{cccc}
\hline & standard SSFP & sparse_HB & sparse_FB \\
\hline EDV [ml] & $128(43)$ & $120(39)$ & $127(38)$ \\
ESV [ml] & $45(33)$ & $45(25)$ & $49(24)$ \\
SV [ml] & $76(20)$ & $68(20)$ & $72(26)$ \\
EF [\%] & $61(11)$ & $60(10)$ & $59(11)$ \\
\hline
\end{tabular}

SSFP, steady-state free precession sequence; sparse, compressed sensing sequence; HB, breath-hold; FB, free breathing; EDV, end-diastolic volume; ESV, end-systolic volume; SV, stroke volume; EF, ejection fraction.

Table 3. Results of the Bland-Altman analysis comparing the volumetric values of the standard SSFP sequence with both sparse sequences.

\begin{tabular}{ccccc}
\hline & standard SSFP vs. & bias & SD & $95 \%$-CI \\
\hline \multirow{2}{*}{ EDV [ml] } & sparse_HB & 7 & 9 & $-10,24$ \\
& sparse_FB & 0 & 10 & $-19,20$ \\
\multirow{2}{*}{ ESV [ml] } & sparse_HB & 1 & 6 & $-10,12$ \\
& sparse_FB & -4 & 7 & $-18,9$ \\
SV [ml] & sparse_HB & 6 & 8 & $-10,22$ \\
& sparse_FB & 5 & 10 & $-15,24$ \\
EF [\%] & sparse_HB & 2 & 4 & $-6,10$ \\
& sparse_FB & 4 & 5 & $-6,13$ \\
\hline
\end{tabular}

SSFP, steady-state free precession sequence; sparse, compressed sensing cine sequence; HB, breath-hold; FB, free breathing; SD, standard deviation; 95\%-CI, 95\%-confidence interval; EDV, end-diastolic volume; ESV, end-systolic volume; SV, stroke volume; EF, ejection fraction.
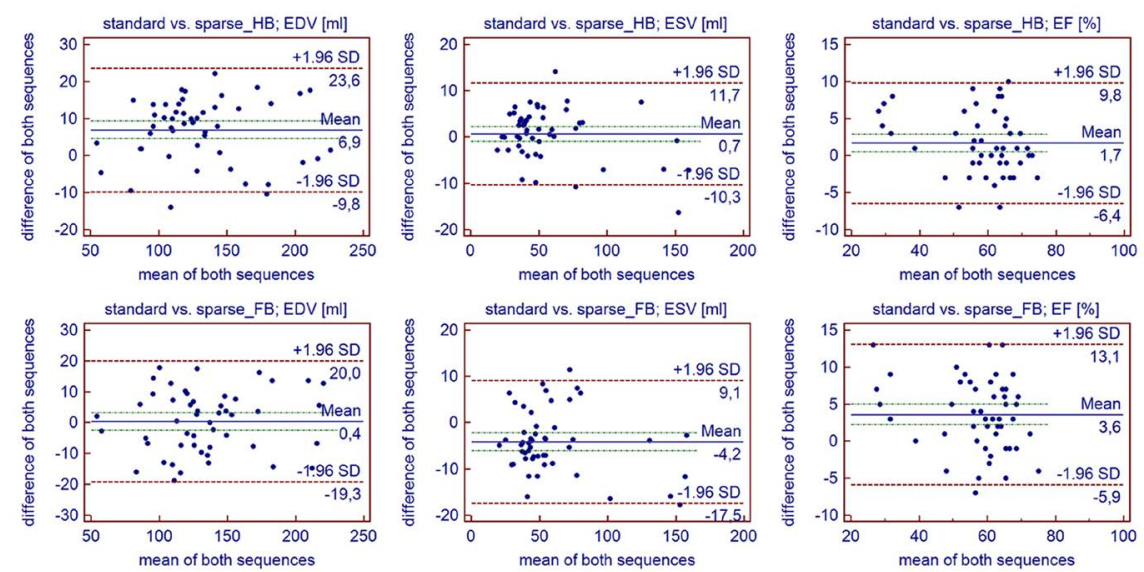

Figure 3. Bland-Altman plots demonstrate a good agreement between the volumetric values derived from the standard SSFP sequence and both sparse sequences.

\section{Discussion}

In this study we could demonstrate for the first time that not only our compressed sensing CMR sequence acquired in breath-hold technique, but also our compressed sensing CMR sequence during free breathing allowed reliable regional left ventricular function assessment. This result is in line with the findings 
of Allen et al. who compared an iteratively reconstructed k-t under sampled breath-hold SENSE cine sequence with a conventional breath-hold SSFP cine sequence based on GRAPPA (acceleration factor, 2) with respect to RWMA detection in 20 patients and in 9 healthy volunteers [7]. They rated the RWMA qualitatively as dichotomous variable (RWMA present or absent) and found a good to excellent agreement between both CMR sequences with Cohens kappa ranging from 0.61 to 0.77 . Similar results were described by Lin et al. who investigated the detect ability of RWMA using a compressed sensing CMR sequence with high spatial and temporal resolution in breath-hold technique (acceleration factor: 8; temporal resolution: $30 \mathrm{~ms}$; in-plane resolution: $1.5 \times 1.5 \mathrm{~mm}^{2}$ ) in comparison to a conventional SSFP sequence (acceleration factor: 2; temporal resolution: $30 \mathrm{~ms}$; in plane resolution $1.25 \times 1.25 \mathrm{~mm}^{2}$ ) in 50 patients [24]. They evaluated the RWMA quantitatively by use of the dedicated post processing software cvi42 (Circle Cardiovascular Imaging, Canada) and reported a strong correlation for RWMA detection between both sequences (Pearson correlation: r, 0.87; $p$ value $<0.001)$, thus, stated that their investigated compressed sensing sequence might replace the conventional time-consuming multiple breath-hold sequences. Based on our results, we totally agree with Lin et al. and believe that the faster compressed sensing CMR sequences, which were proven to reliable detect RWMA, have the potential to replace standard cine SSFP imaging in clinical routine for the assessment of regional and global LV function. Moreover, compressed sensing cine imaging is extremely interesting for high-dose dobutamine stress CMR. The latter is often hampered by motion artifacts caused by dobutamine induced tachycardia. And these motion artifacts might considerably be reduced by the faster CMR data acquisition of our tested compressed sensing sequences. This anticipated improvement by use of compressed sensing sequences in high-dose dobutamine stress CMR should be investigated in further studies.

Beyond the discussed studies, no other compressed sensing CMR sequence study dealt with RWMA detection, and to the best of our knowledge our study is the first investigating a compressed sensing CMR sequence during free breathing with respect to RWMA detection. And given that many patients undergoing CMR suffer from shortness of breath, CMR data acquisition during free breathing improves not only CMR acceptance by the patient but also patient's comfort.

Regarding the global left ventricular function, only small, not relevant differences in left ventricular values were found between the standard SSFP sequence and both compressed sensing CMR sequences. For the sparse_HB sequence slightly lower EDV, SV, and EF values were found which might be caused by an insufficient capture of the end-diastole [10]. For the sparse_FB sequences lightly higher ESV and consecutively lower EF values were found. But overall a sufficient agreement between the standard SSFP sequence and both sparse sequences was found which is in accordance to recently published studies where a sufficient to excellent agreement between the investigated sparse and reference se- 
quences were reported [10] [12] [13].

Our study is not without limitations. First, we analyzed the RWMA exclusively visually. Although this is common practice, accuracy might benefit from a quantitative RWMA analysis. Second, only a limited number of patients/cardiac segments with RWMA were included, which was due to our unselected patient cohort.

\section{Conclusion}

In conclusion, compressed sensing cine imaging of the left ventricle acquired either during breath-hold or during free breathing allows the reliable detection of regional wall motion abnormalities. Thus, these fast cine sequences can alternatively be used for the assessment of LV function.

\section{Acknowledgements}

The authors thank Marcel Gratz for his technical assistance and fruitful discussions.

\section{Conflict of Interest}

The SPARSE-SENSE sequence prototype was provided by Siemens Healthcare $\mathrm{GmbH}$, Erlangen, Germany. The authors declare that there is no conflict of interest to this article.

\section{References}

[1] Grothues, F., Smith, G.C., Moon, J.C., Bellenger, N.G., Collins, P., Klein, H.U. and Pennell, D.J. (2002) Comparison of Interstudy Reproducibility of Cardiovascular Magnetic Resonance with Two-Dimensional Echocardiography in Normal Subjects and in Patients with Heart Failure or Left Ventricular Hypertrophy. American Journal of Cardiology, 90, 29-34. https://doi.org/10.1016/S0002-9149(02)02381-0

[2] McMurray, J.J., Adamopoulos, S., Anker, S.D., Auricchio, A., Bohm, M., Dickstein, K., Falk, V., Filippatos, G., Fonseca, C., Gomez-Sanchez, M.A., Jaarsma, T., Kober, L., Lip, G.Y., Maggioni, A.P., Parkhomenko, A., Pieske, B.M., Popescu, B.A., Ronnevik, P.K., Rutten, F.H., Schwitter, J., Seferovic, P., Stepinska, J., Trindade, P.T., Voors, A.A., Zannad, F., Zeiher, A. and Guidelines, E.S.C.C.f.P. (2012) ESC Guidelines for the Diagnosis and Treatment of Acute and Chronic Heart Failure 2012: The Task Force for the Diagnosis and Treatment of Acute and Chronic Heart Failure 2012 of the European Society of Cardiology. Developed in Collaboration with the Heart Failure Association (HFA) of the ESC. European Heart Journal, 33, 1787-1847. https://doi.org/10.1093/eurheartj/ehs104

[3] Brignole, M., Auricchio, A., Baron-Esquivias, G., Bordachar, P., Boriani, G., Breithardt, O.A., Cleland, J., Deharo, J.C., Delgado, V., Elliott, P.M., Gorenek, B., Israel, C.W., Leclercq, C., Linde, C., Mont, L., Padeletti, L., Sutton, R., Vardas, P.E., Guidelines, E.S.C.C.F.P., Zamorano, J.L., Achenbach, S., Baumgartner, H., Bax, J.J., Bueno, H., Dean, V., Deaton, C., Erol, C., Fagard, R., Ferrari, R., Hasdai, D., Hoes, A.W., Kirchhof, P., Knuuti, J., Kolh, P., Lancellotti, P., Linhart, A., Nihoyannopoulos, P., Piepoli, M.F., Ponikowski, P., Sirnes, P.A., Tamargo, J.L., Tendera, M., Torbicki, A., Wijns, W., Windecker, S., Document, R., Kirchhof, P. Blomstrom-Lundqvist, C., Badano, L.P., Aliyev, F., Bansch, D., Baumgartner, H., 
Bsata, W., Buser, P., Charron, P., Daubert, J.C., Dobreanu, D., Faerestrand, S., Hasdai, D., Hoes, A.W., Le Heuzey, J.Y., Mavrakis, H., McDonagh, T., Merino, J.L., Nawar, M.M., Nielsen, J.C., Pieske, B., Poposka, L., Ruschitzka, F., Tendera, M., Van Gelder, I.C. and Wilson, C.M. (2013) 2013 ESC Guidelines on Cardiac Pacing and Cardiac Resynchronization Therapy: The Task Force on Cardiac Pacing and Resynchronization Therapy of the European Society of Cardiology (ESC). Developed in Collaboration with the European Heart Rhythm Association (EHRA). European Heart Journal, 34, 2281-2329. https://doi.org/10.1093/eurheartj/eht150

[4] Lustig, M., Donoho, D. and Pauly, J.M. (2007) Sparse MRI: The Application of Compressed Sensing for Rapid MR Imaging. Magnetic Resonance in Medicine, 58, 1182-1195. https://doi.org/10.1002/mrm.21391

[5] Vincenti, G., Piccini, D., Monney, P., Chaptinel, J., Rutz, T., Coppo, S., Zenge, M.O., Schmidt, M., Nadar, M.S., Wang, Q., Chevre, P., Stuber, M. and Schwitter, J. (2013) Preliminary Experiences with Compressed Sensing Multi-Slice Cine Acquisitions for the Assessment of Left Ventricular Function: CV_sparse WIP. MAGNETOM Flash, 5, 108-116.

[6] Feng, L., Srichai, M.B., Lim, R.P., Harrison, A., King, W., Adluru, G., Dibella, E.V., Sodickson, D.K., Otazo, R. and Kim, D. (2013) Highly Accelerated Real-Time Cardiac Cine MRI Using k-t SPARSE-SENSE. Magnetic Resonance in Medicine, 70, 64-74. https://doi.org/10.1002/mrm.24440

[7] Allen, B.D., Carr, M., Botelho, M.P., Rahsepar, A.A., Markl, M., Zenge, M.O., Schmidt, M., Nadar, M.S., Spottiswoode, B., Collins, J.D. and Carr, J.C. (2016) Highly Accelerated Cardiac MRI Using Iterative SENSE Reconstruction: Initial Clinical Experience. The International Journal of Cardiovascular Imaging, 32, 955-963. https://doi.org/10.1007/s10554-016-0859-3

[8] Vincenti, G., Monney, P., Chaptinel, J., Rutz, T., Coppo, S., Zenge, M.O., Schmidt, M., Nadar, M.S., Piccini, D., Chevre, P., Stuber, M. and Schwitter, J. (2014) Compressed Sensing Single-Breath-Hold CMR for Fast Quantification of LV Function, Volumes, and Mass. JACC Cardiovasc Imaging, 7, 882-892.

https://doi.org/10.1016/j.jcmg.2014.04.016

[9] Goebel, J., Nensa, F., Schemuth, H.P., Maderwald, S., Gratz, M., Quick, H.H., Schlosser, T. and Nassenstein, K. (2016) Compressed Sensing Cine Imaging with High Spatial or High Temporal Resolution for Analysis of Left Ventricular Function. Journal of Magnetic Resonance Imaging. https://doi.org/10.1002/jmri.25162

[10] Kido, T., Kido, T., Nakamura, M., Watanabe, K., Schmidt, M., Forman, C. and Mochizuki, T. (2016) Compressed Sensing Real-Time Cine Cardiovascular Magnetic Resonance: Accurate Assessment of Left Ventricular Function in a Single-Breath-Hold. Journal of Cardiovascular Magnetic Resonance, 18, 50. https://doi.org/10.1186/s12968-016-0271-0

[11] Kido, T., Kido, T., Nakamura, M., Watanabe, K., Schmidt, M., Forman, C. and Mochizuki, T. (2017) Assessment of Left Ventricular Function and Mass on Free-Breathing Compressed Sensing Real-Time Cine Imaging. Circulation Journal, 81, 1463-1468. https://doi.org/10.1253/circj.CJ-17-0123

[12] Camargo, G.C., Erthal, F., Sabioni, L., Penna, F., Strecker, R., Schmidt, M., Zenge, M.O., Lima, R.S. and Gottlieb, I. (2017) Real-Time Cardiac Magnetic Resonance cine Imaging with Sparse Sampling and Iterative Reconstruction for Left-Ventricular Measures: Comparison with Gold-Standard Segmented Steady-State Free Precession. Journal of Magnetic Resonance Imaging, 38, 138-144.

https://doi.org/10.1016/j.mri.2017.01.002

[13] Sudarski, S., Henzler, T., Haubenreisser, H., Dosch, C., Zenge, M.O., Schmidt, M., 
Nadar, M.S., Borggrefe, M., Schoenberg, S.O. and Papavassiliu, T. (2017) Free-Breathing Sparse Sampling Cine MR Imaging with Iterative Reconstruction for the Assessment of Left Ventricular Function and Mass at 3.0 T. Radiology, 282, 74-83. https://doi.org/10.1148/radiol.2016151002

[14] Underwood, S.R., Rees, R.S., Savage, P.E., Klipstein, R.H., Firmin, D.N., Fox, K.M., Poole-Wilson, P.A. and Longmore, D.B. (1986) Assessment of Regional Left Ventricular Function by Magnetic Resonance. British Heart Journal, 56, 334-340. https://doi.org/10.1136/hrt.56.4.334

[15] Gardner, B.I., Bingham, S.E., Allen, M.R., Blatter, D.D. and Anderson, J.L. (2009) Cardiac Magnetic Resonance versus Transthoracic Echocardiography for the Assessment of Cardiac Volumes and Regional Function after Myocardial Infarction: An Intrasubject Comparison Using Simultaneous Intrasubject Recordings. Cardiovascular Ultrasound, 7, 38. https://doi.org/10.1186/1476-7120-7-38

[16] Nagel, E., Lehmkuhl, H.B., Bocksch, W., Klein, C., Vogel, U., Frantz, E., Ellmer, A., Dreysse, S. and Fleck, E. (1999) Noninvasive Diagnosis of Ischemia-Induced Wall Motion Abnormalities with the Use of High-Dose Dobutamine Stress MRI: Comparison with Dobutamine Stress Echocardiography. Circulation, 99, 763-770. https://doi.org/10.1161/01.CIR.99.6.763

[17] Paetsch, I., Jahnke, C., Wahl, A., Gebker, R., Neuss, M., Fleck, E. and Nagel, E. (2004) Comparison of Dobutamine Stress Magnetic Resonance, Adenosine Stress Magnetic Resonance, and Adenosine Stress Magnetic Resonance Perfusion. Circulation, 110, 835-842. https://doi.org/10.1161/01.CIR.0000138927.00357.FB

[18] Heilmaier, C., Bruder, O., Meier, F., Jochims, M., Forsting, M., Sabin, G.V., Barkhausen, J. and Schlosser, T.W. (2009) Dobutamine Stress Cardiovascular Magnetic Resonance Imaging in Patients after Invasive Coronary Revascularization with Stent Placement. Acta Radiologica, 50, 1134-1141. https://doi.org/10.3109/02841850903216692

[19] Cerqueira, M.D., Weissman, N.J., Dilsizian, V., Jacobs, A.K., Kaul, S., Laskey, W.K., Pennell, D.J., Rumberger, J.A., Ryan, T. and Verani, M.S., American Heart Association Writing Group on Myocardial Segmentation and Registration for Cardiac Imaging (2002) Standardized Myocardial Segmentation and Nomenclature for Tomographic Imaging of the Heart: A Statement for Healthcare Professionals from the Cardiac Imaging Committee of the Council on Clinical Cardiology of the American Heart Association. Circulation, 105, 539-542.

https://doi.org/10.1161/hc0402.102975

[20] Maceira, A.M., Prasad, S.K., Khan, M. and Pennell, D.J. (2006) Normalized Left Ventricular Systolic and Diastolic Function by Steady State Free Precession Cardiovascular Magnetic Resonance. Journal of Cardiovascular Magnetic Resonance, 8 , 417-426. https://doi.org/10.1080/10976640600572889

[21] Papavassiliu, T., Kuhl, H.P., Schroder, M., Suselbeck, T., Bondarenko, O., Bohm, C.K., Beek, A., Hofman, M.M. and van Rossum, A.C. (2005) Effect of Endocardial Trabeculae on Left Ventricular Measurements and Measurement Reproducibility at Cardiovascular MR Imaging. Radiology, 236, 57-64. https://doi.org/10.1148/radiol.2353040601

[22] Nassenstein, K., Eberle, H., Maderwald, S., Jensen, C.J., Heilmaier, C., Schlosser, T. and Bruder, O. (2010) Single Breath-Hold Magnetic Resonance Cine Imaging for Fast Assessment of Global and Regional Left Ventricular Function in Clinical Routine. European Radiology, 20, 2341-2347. https://doi.org/10.1007/s00330-010-1827-2

[23] Landis, J.R. and Koch, G.G. (1977) The Measurement of Observer Agreement for 
Categorical Data. Biometrics, 33, 159-174. https://doi.org/10.2307/2529310

[24] Lin, A.C.W., Strugnell, W., Riley, R., Schmitt, B., Zenge, M., Schmidt, M., Morris, N.R. and Hamilton-Craig, C. (2017) Higher Resolution Cine Imaging with Compressed Sensing for Accelerated Clinical Left Ventricular Evaluation. Journal of Magnetic Resonance Imaging, 45, 1693-1699. https://doi.org/10.1002/jmri.25525 